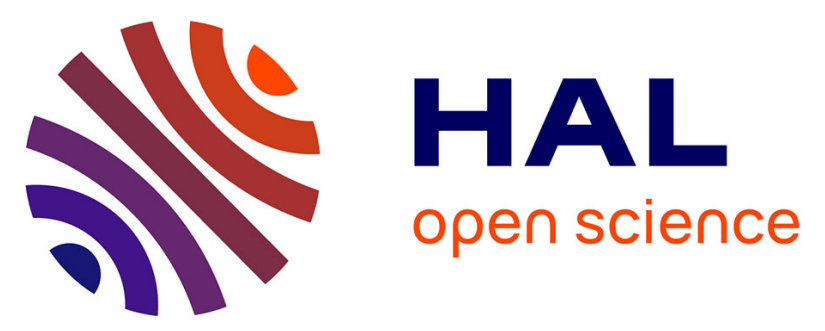

\title{
Enjeux relationnels et limites d'application des directives anticipées : étude qualitative exploratoire auprès de médecins
}

Béatrice Eon, Merlin Paul, Patrice Cannone, Pierre Le Coz, Lionel Dany

\section{To cite this version:}

Béatrice Eon, Merlin Paul, Patrice Cannone, Pierre Le Coz, Lionel Dany. Enjeux relationnels et limites d'application des directives anticipées: étude qualitative exploratoire auprès de médecins. Revue internationale de soins palliatifs, 2017, Défis et complexité, 32 (3), pp.69-74. 10.3917/inka.173.0069 . hal-01672830

\section{HAL Id: hal-01672830 \\ https://hal-amu.archives-ouvertes.fr/hal-01672830}

Submitted on 27 Dec 2017

HAL is a multi-disciplinary open access archive for the deposit and dissemination of scientific research documents, whether they are published or not. The documents may come from teaching and research institutions in France or abroad, or from public or private research centers.
L'archive ouverte pluridisciplinaire HAL, est destinée au dépôt et à la diffusion de documents scientifiques de niveau recherche, publiés ou non, émanant des établissements d'enseignement et de recherche français ou étrangers, des laboratoires publics ou privés. 


\section{ENJEUX RELATIONNELS ET LIMITES D'APPLICATION DES DIRECTIVES ANTICIPEES : ETUDE QUALITATIVE EXPLORATOIRE AUPRES DE MEDECINS}

Béatrice Eon ${ }^{1}$, Merlin Paul ${ }^{2}$, Patrice Cannone ${ }^{3}$, Pierre Le Coz ${ }^{1}$, Lionel Dany ${ }^{1,2,4}$

\footnotetext{
${ }^{1}$ Aix Marseille Univ, CNRS, EFS, ADES, Marseille, France

${ }^{2}$ Aix Marseille Univ, LPS, Aix-en-Provence, France

${ }^{3}$ APHM, Nord, Service d'Oncologie Multidisciplinaire et Innovations Thérapeutiques, Marseille, France

${ }^{4}$ APHM, Timone, Service d'Oncologie Médicale, Marseille, France
}

\section{Référence}

Eon, B., Paul, M., Cannone, P., Le Coz, P., \& Dany, L. (2017). Enjeux relationnels et limites d'application des directives anticipées : étude qualitative exploratoire auprès de médecins. Revue Internationale de Soins Palliatifs, 32 (3), 69-74. 
Résumé : Les médecins et les directives anticipées - Dans le contexte du développement des directives anticipées (DA) en France depuis 2016, notre étude avait pour objectif d'étudier les connaissances, représentations et modalités d'intégration de ce dispositif auprès de médecins. Nous avons conduit une recherche qualitative monocentrique par entretiens de recherche auprès de médecins $(n=12)$. Une analyse de contenu thématique a été réalisée sur l'ensemble des entretiens. L'analyse de contenu des entretiens a mis en évidence une connaissance relative des DA, des réserves quant au rôle des médecins par rapport à ce dispositif et un ensemble de limites perçues concernant leur mise en œuvre (complexité des situations de fin de vie; impact sur la relation de soin ; répercussion psychologique ; incertitude; caractère "changeant» des DA; culture des services de soins). Des limites d'ordre psychosocial expliquent en partie les réticences concernant ce dispositif et contribuent à une mise en question de sa «légitimité » ou « utilité ». L'enjeu du développement des connaissances sur les DA doit s'accompagner d'une réflexion sur les conditions psychosociales de son acceptabilité et de son intégration dans les pratiques et la relation soignantes.

Summary: Physicians and advance directives - In the context of the development of advance directives (AD) in France, our study aimed to study the knowledge, representations and modalities of integration of this device for physicians. We conducted a qualitative research by interviews with a sample of physicians $(n=12)$. A thematic content analysis was carried out on all the interviews. The analysis of the content of the interviews revealed a relative knowledge of the $A D$, some reservations concerning the role of doctors in relation to this device and a set of perceived limits on their implementation (the complexity of end-of-life situations, the impact on care relation, the psychological repercussion, uncertainty, the "changing" nature of $A D$, the culture of care services). Psychosocial limitations partly explain the reluctance of this device and contribute to a questioning of its "legitimacy" or "utility". The challenge of developing knowledge about $A D$ must be accompanied by a reflection on the psychosocial conditions of its acceptability and its integration into care practices and relation.

Mots-clés : Directives anticipées - Médecins - Fin de vie - Communication médecin-patient

Key-words: Advance directives - Physicians - End-of-life - Patient-doctor communication

Correspondance: Pr. Lionel Dany, Aix-Marseille Université, Laboratoire de Psychologie Sociale, Maison de la Recherche SCHUMAN, 29 avenue R. Schuman - 13628 Aix-en-Provence, France. Courriel: Lionel.Dany@univ-amu.fr 


\section{INTRODUCTION}

Introduite pour la première fois en France par la loi du 22 avril 2005, les directives anticipées (DA) reflètent un investissement du pouvoir législatif concernant la prise en charge en fin de vie. [1] Les directives anticipées sont l'expression écrite, par avance, des souhaits d'une personne (malade ou non) de limiter ou arrêter un ou des traitements qui pourraient lui être proposés alors qu'elle ne serait plus en mesure de s'exprimer par elle-même et d'y consentir ou non. [2] La loi $N^{\circ} 2016-87$ du 2 février 2016, créant de nouveaux droits en faveur des malades et des personnes en fin de vie, a apporté des modifications substantielles aux DA. [3] La principale évolution concerne leur caractère désormais contraignant et non plus seulement consultatif hormis deux situations : l'urgence vitale et le caractère manifestement inapproprié de leur contenu à la situation. Initialement, les DA étaient envisagées comme l'expression de souhaits et sont devenues actuellement l'expression d'une volonté. Rédiger une DA aujourd'hui, implique une demande de respect stricto-sensu de celle-ci ou de prise en considération par le professionnel de santé. [4]

Les DA disposent a priori de certains avantages. Leur mise en œuvre peut contribuer à anticiper les situations aiguës prévisibles pour définir des conduites à tenir en adéquation avec les volontés du patient dans le but d'éviter des traitements auxquels il n'aurait pas consenti ; permettre de disposer d'informations utiles du point de vue du patient dans le cadre des délibérations collégiales; d'améliorer l'accompagnement en fin de vie; de contribuer à une prise en compte « réelle » des souhaits du patient concernant sa fin de vie ; de favoriser l'acceptation des décisions auprès de la famille et des proches voire pour prévenir des situations de contentieux.

En dépit de ses apports, force est de constater que les DA restent peu connues ou peu mises en œuvre. [5] En France, selon l'enquête La fin de vie en France réalisée par l'Ined en 2010, seuls $2,5 \%$ des patients concernés auraient rédigé leurs directives anticipées. [6] Diverses études portant sur les taux de recours aux DA pour les personnes en bonne santé comme pour les personnes atteintes d'une maladie aiguë, chronique ou encore létale, montrent que ce recours se situe entre 5 et 25\%. [7-12] Ces taux de recours aux DA n'ont 
pas connu d'évolution particulière malgré les attitudes en majorité favorables exprimées visà-vis de ce dispositif. [13]

Avec les DA, les patients se trouvent dans une position nouvelle dans la trajectoire de soins car ils peuvent infléchir et/ou orienter, par leur choix anticipé, le projet de soin et, d'une certaine manière, "l'imposer » aux soignants. Ce dispositif comporte, de fait, un certain nombre d'enjeux pour la relation thérapeutique et la prise en charge des patients en situation de fin de vie et interroge les perceptions et représentations des médecins face à cette nouvelle configuration.

Certains travaux montrent que bien qu'ils soient favorables aux DA, les médecins y sont moins favorables que leurs patients et qu'ils les utilisent rarement. [14,15] Par ailleurs, lorsque les DA des patients sont en conflit avec leurs opinions, les médecins seraient tentés de privilégier ce qu'ils considèrent comme relevant du bénéfice du patient par rapport à son autonomie décisionnelle. [16] Ce constat semble plus marqué en France que dans d'autre pays, à la tradition plus « libérale » comme l'Angleterre. [17]

Dans un contexte de développement du dispositif des DA, il nous semble important d'étudier, chez les médecins, la façon dont ils appréhendent la mise en œuvre pratique des DA (réelle et/ou perçue), et ce, en l'articulant avec leurs préférences relatives au rôle des patients dans ce contexte. Dans une perspective compréhensive et socio-contextuelle, l'étude des représentations et pratiques des médecins à l'égard des DA nous a semblé particulièrement utile pour éclairer les logiques socio-professionnelles qui peuvent faciliter ou limiter le recours aux DA.

L'objectif de cette étude était d'étudier les connaissances, représentations et modalités d'intégration (i.e. inscription perçue du dispositif dans les pratiques professionnelles) des DA auprès de médecins impliqués dans la prise en charge de patients susceptibles de ne pas pouvoir exprimer leurs décisions sur la limitation ou l'arrêt limiter des traitements.

\section{MATERIEL ET METHODES}

Nous avons conduit une recherche qualitative monocentrique par entretiens de recherche 
auprès de médecins exerçant au sein de l'Assistance Publique - Hôpitaux de Marseille (APHM). Le guide d'entretien visait à recueillir les connaissances et significations élaborées par les médecins au sujet des DA: représentations des DA, définitions et expériences personnelles, dernière situation rencontrée, opinions concernant l'application des DA.

La participation à cette recherche a été proposée à des spécialistes en interactions fréquentes avec l'équipe médicale de réanimation.

Au total douze médecins (sur les vingt-quatre contactés) ont acceptés de participer à l'étude. L'échantillon comprend cinq femmes et sept hommes. Les spécialités représentées étaient la cardiologie (4), la néphrologie (2), l'oncologie (2), la neurologie (2), l'oncogériatrie (1) et la gériatrie (1). L'âge moyen des médecins interrogés était de 46 ans (32 à 57 ans). La durée moyenne des entretiens était de 66 minutes (de 49 à $74 \mathrm{mn}$ ).

Les entretiens ont été intégralement retranscrits et ont fait l'objet d'une analyse de contenu. $[18,19]$ Cette analyse a été effectuée à un niveau vertical (chaque entretien) et à un niveau horizontal (l'ensemble des entretiens). Après une phase d'immersion et de familiarisation, nous avons identifié et sélectionné des unités de sens [20] en lien avec notre problématique de recherche afin de permettre l'inférence des connaissances. Les entretiens ont été réalisés dans un cadre éthique basé sur plusieurs points clés : garantie de l'anonymat des personnes interrogées, participation volontaire de chaque personne interrogée, possibilité pour chaque personne interviewée de consulter les données. Le projet a reçu un avis favorable de la Commission d'Ethique de la Société de Réanimation de Langue Française (CE SRLF15-53).

\section{RESULTATS}

L'analyse de contenu des entretiens a permis l'émergence de différents thèmes relatifs à la connaissance perçue des DA, les réserves énoncées par les médecins par rapport à ce dispositif, enfin, une dernière thématique recouvre les différentes limites perçues concernant la mise en œuvre des DA. 


\section{La connaissance perçue des directives anticipées}

Les médecins interviewés expriment une connaissance incomplète voire erronée des DA (ex. durée de validité, circonstances de leur prise en compte, rédaction systématique devant témoins). La méconnaissance de la Loi Leonetti de 2005 et du décret relatif aux DA est reconnue par les médecins. Lorsqu'ils décrivent les $D A$, les médecins se réfèrent à ce que l'on peut rapprocher du principe du respect de l'autonomie et aux volontés en fin de vie afin d'éviter l'acharnement thérapeutique. Les médecins se retrouvent, d'une certaine manière, dans un contexte qui les rend responsables d'une conduite à tenir.

« [Les DA] C'est décider pour soi-même ce qui pourrait, ce que l'on veut qu'il se passe quand on n'a plus les capacités mentales ou physiques de prendre des décisions soi-même » (E2, neurologue)

« L'art et la manière de finir sa vie » (E11, néphrologue)

\section{Les réserves des médecins}

Une autre thématique importante concerne ce que nous avons nommé « les réserves » des médecins. Ces réserves des médecins s'expriment lorsqu'ils doivent clarifier ou circonscrire leur rôle possible dans la mise en œuvre de DA. De fait, les avis exprimés reflètent un questionnement sur la pertinence du moment, le lieu opportun, la nature de la maladie, la disponibilité en temps voire la "rentabilité » d'une activité centrée sur l'information ou la réflexion. Certains expriment le fait de ne pas se sentir totalement concernés (en référence souvent à leur spécialité). Mais ces réserves portent également sur la liberté réelle qui peut être laissée au patient.

« Je ne vois pas mon rôle médical comme quelqu'un qui peut aider à rédiger des directives anticipées... ça devrait pas être des professionnels, enfin ... pas des médecins qui s'occupent de la pathologie » (E11, néphrologue)

« Je pense qu'à un moment il faut quand même avoir ses convictions et quand même les orienter, on peut pas leur dire "à vous de choisir " non plus parce qu'ils ne savent pas eux...» $(E 9$, oncogériatre $)$ 


\section{Les limites perçues pour la mise en œuvre des directives anticipées}

Un certain nombre de limites ont pu être identifiées dans le discours des médecins. Ces limites peuvent être catégorisées en fonction de leur « nature » (cf. tableau 1). La première catégorie concerne le caractère complexe des situations de fin de vie. Cette complexité renvoie d'une part, à la présence d'un grand nombre de paramètres dans la situation et, d'autre part, à l'imprévisibilité de ces paramètres. La deuxième catégorie porte plus spécifiquement sur l'impact potentiel de la contrainte légale que suppose les DA sur la relation entre le soignant et le patient. La mise en œuvre des DA est vue, à ce titre, comme un élément "étranger » pouvant se substituer à une relation thérapeutique envisagée comme plus «humaine ». La troisième catégorie de limites concerne les répercussions potentielles - en particulier psychologique - sur le patient de l'évocation ou de la proposition de mise en œuvre des DA. La quatrième catégorie renvoie au contexte d'incertitude qui accompagne le pronostic médical et les aléas thérapeutiques. Cette catégorie concerne également l'incertitude relative au jugement du patient sur sa situation et sur ses DA. La cinquième catégorie concerne le caractère "changeant" des DA chez les patients. Autrement dit, il s'agit d'une limite qui porte sur le degré de certitude concernant les attentes et décisions du patient qui peuvent se modifier ou être remises en cause selon sa trajectoire personnelle.

Enfin, la sixième et dernière catégorie, renvoie à ce qui relève, selon les médecins, des limites propres à la culture des services de soins. Ce type de limite porte, d'une part, sur les spécificités de certaines spécialités (et par extension de certains services) qui peuvent être dans une logique de prolongation des soins, d'autre part, sur une caractéristique plus générale qui concerne les possibilités offertes par la science médicale qui permet - dans certains contextes (tel qu'un centre hospitalier universitaire) - de continuer à proposer des options ou opportunités thérapeutiques retardant, de fait, la pertinence de mettre en œuvre des DA. 
Tableau1. Limites évoquées par les médecins pour la mise en œuvre des directives anticipées

\begin{tabular}{|c|c|}
\hline Limites & Extraits \\
\hline $\begin{array}{c}\text { Des situations } \\
\text { complexes/inadaptées }\end{array}$ & $\begin{array}{l}\text { "Ce sont des situations qui sont complexes et en fait souvent avec de } \\
\text { multiples paramètres à prendre en compte " (E3, gériatre) } \\
\text { "Les phénomènes qui amènent à la fin de vie en cardiologie sont } \\
\text { souvent brutaux et ce ne sont pas des déchéances progressives, [ou] des } \\
\text { états végétatifs qui amènent à se poser ce genre de questions [celles } \\
\text { relatives aux DA], à les anticiper " (E12, cardiologue) }\end{array}$ \\
\hline $\begin{array}{c}\text { Caractère substitutif à } \\
\text { la relation }\end{array}$ & $\begin{array}{l}\text { "Je me dit qu'une procédure risque de déshumaniser quelque chose qui } \\
\text { demande beaucoup d'humain " (E3, gériatre) } \\
\text { "Je ne sais pas si ça serait approprié, je trouve que c'est un domaine } \\
\text { tellement difficile, qu'introduire des lois dedans (...) je ne sais pas si c'est } \\
\text { pas excessif et inadapté par rapport à des problèmes qui se règlent sur le } \\
\text { plan humain entre l'équipe médicale, la famille " (E8, cardiologue) }\end{array}$ \\
\hline $\begin{array}{l}\text { Impact psychologique } \\
\text { sur le patient }\end{array}$ & $\begin{array}{l}\text { "Comment l'amener dans la discussion, d'une façon qui n'est pas trop } \\
\text { traumatisante? " (E1, néphrologue) } \\
\text { "Dès que je sens qu'il y a de l'anxiété derrière, du patient, de la famille } \\
\text { en général, je suis un peu hypocrite, j'essaye de m'adapter au profil } \\
\text { psychologique du patient et on ne leur dit pas forcément les choses très } \\
\text { crûment comme ça " (E8, cardiologue) }\end{array}$ \\
\hline Incertitude & $\begin{array}{l}\text { "Je vais être, en fait, embêté parce que je ne peux pas répondre de } \\
\text { façon claire, scientifique et surtout certaine " (E6, cardiologue) } \\
\text { "Moi ça interfère pas mal parce que souvent je n'ai pas toujours la } \\
\text { réponse [...] l'incertitude oui ça fait partie de ma pratique " (E9, } \\
\text { oncogériatre) } \\
\text { "On a du mal à croire que le patient est capable à } 100 \% \text { de faire des } \\
\text { directives anticipées qui vont être justes dans un sens comme dans } \\
\text { l'autre " (E12, cardiologue) }\end{array}$ \\
\hline $\begin{array}{l}\text { Caractère changeant } \\
\text { des directives }\end{array}$ & $\begin{array}{l}\text { "Pas sûre qu'une personne qui est en fin de vie a la même vision de la } \\
\text { qualité de vie que quelqu'un qui est en pleine forme et qui décide à ce } \\
\text { moment-là ce qu'il en sera de sa fin de sa vie " (E2, neurologue) } \\
\text { « II [un patient] m'avait donné des DA et il a changé d'avis. En fait non, s'il } \\
\text { a un problème il veut être réanimé et il veut continuer à vivre " (E10, } \\
\text { neurologue) }\end{array}$ \\
\hline $\begin{array}{l}\text { Idéologie/culture du } \\
\text { service/spécialité }\end{array}$ & $\begin{array}{l}\text { "Ça tient beaucoup à la spécialité, à la mentalité. Je crois qu'on est une } \\
\text { spécialité un peu particulière dans le sens où on a tellement l'habitude de } \\
\text { penser qu'on va toujours pouvoir continuer [...] ça tient à l'idée que si } \\
\text { l'organe qui nous intéresse ne fonctionne plus on continue toujours " (E1, } \\
\text { néphrologue) } \\
\text { "On a la fâcheuse tendance de vouloir toujours être trop optimiste et à } \\
\text { vouloir toujours essayer de proposer quelque chose de supplémentaire } \\
\text { aux patients ou à laisser une opportunité supplémentaire " (E12, } \\
\text { cardiologue) }\end{array}$ \\
\hline
\end{tabular}




\section{DISCUSSION}

Face aux enjeux réactualisés des directives anticipées dans le contexte français, l'objectif de cette étude était d'étudier les modalités de connaissances professionnelles et psychosociales (représentations, croyances) qui accompagnent les réflexions et pratiques des soignants vis-à-vis des directives anticipées. Cette étude qualitative exploratoire a permis d'identifier un ensemble de facteurs pouvant interférer avec la mise en œuvre des DA en situation de soin chez les médecins.

Un premier élément de discussion concerne la connaissance déclarée par les médecins au sujet des DA. Cette connaissance incomplète ou parfois erronée se retrouve dans d'autres études réalisées auprès de soignants et également dans la population générale. [7, 21] L'enjeu d'une connaissance « objective » du dispositif des DA est un enjeu important voire pourrait constituer une condition nécessaire (mais pas suffisante) à sa mise en œuvre (i.e. cadre et modalités d'application). Ce résultat pointe, de fait, la nécessité de former les soignants (formation initiale et continue) sur les DA en lien avec les enseignements relevant de la fin de vie et des soins palliatifs.

II nous semble également important de souligner que l'absence de connaissances objectives ne constituent pas a priori un obstacle à la mise en œuvre d'un dispositif tel que les DA. II faut prendre en compte d'autres formes de connaissances - notamment sociales - pour comprendre les processus qui interviennent sur les pratiques effectives et les intentions comportementales des médecins voire sur les formes de rationalisations des conduites professionnelles. Autrement dit, il semble que se soient davantage les réticences exprimées qui agissent sur les pratiques.

Les données issues des entretiens font apparaître un enchevêtrement complexe de facteurs de diverses natures qui peuvent concourir in fine à limiter, d'une part, l'appréhension de l'utilité et/ou de la légitimité des DA, d'autre part, le recours à celles-ci. Ces facteurs sont d'ordre organisationnel (gestion des contraintes structurelles de l'activité de soin), 
institutionnel (articulation et légitimité des activités au sein de l'institution de soin), relationnel (impact, contenu et forme de la relation avec les patients), identitaire (rôle et fonctions des soignants par rapport aux DA, identité des spécialités médicales et des services de soin).

Ces résultats pointent, selon nous, qu'une démarche qui pourrait être appréhendée dans les " strictes » dimensions du colloque singulier entre un médecin et un patient, recouvre au final tout un ensemble d'enjeux qui transcendent cette composante. Les différentes catégories de limites mises en évidence (cf. tableau 1), comme les réserves exprimées par les médecins quant à leurs rôles, permettent de saisir cette complexité des modèles décisionnels qui s'actualisent dans la situation thérapeutique. [22]

L'inscription des DA dans la situation thérapeutique met en évidence une des caractéristiques essentielles de la relation entre le médecin et le patient, à savoir le passage entre la pensée, la réflexion et la mise en acte de cette pensée ou de cette réflexion. L'inscription des DA dans la situation thérapeutique s'apparente une forme de « confrontation en acte de croyances et de représentations » dans ce contexte particulier du soin. [23] Les DA actualisent des enjeux psychosociaux (rôle des connaissances réelles ou perçues et leurs soubassements socio-représentationnels, élaboration d'une posture sceptique et identification de limites en lien avec des postures et enjeux professionnels) que nous avons tenté d'identifier et de discuter mais d'autres enjeux émergent sur le plan juridique ou politique comme en témoignent les débats sociétaux actuels et les «freins » relevés dans différents travaux et enquêtes. [7, 17, 24-25] Cette situation n'est pas sans rappeler ce que l'on peut observer depuis un grand nombre d'années en France concernant la greffe et le don d'organes. [26]

Par ailleurs, les processus de psychologisation (attribution d'une composante et/ou d'un vécu psychologiques aux patients) constituent une limite importante déjà pointée [27] même si différents travaux rapportent que les discussions relatives aux DA ne génèrent pas le niveau d'anxiété anticipé par les soignants [28].

Un autre enjeu, d'ordre éthique, nous semble devoir être discuté. Les résultats mettent en évidence un certain écart entre les DA telles que se les représentent les médecins (un 
dispositif contraignant qui modifie la relation thérapeutique) et ce qu'elles sont censées « être » (un dispositif facilitant la relation thérapeutique et le respect des choix des patients). Les résultats de l'étude pointent, selon nous, une difficulté relative à l'autonomie (autorégulation libre de l'ingérence des autres) des patients. [29] En effet, du fait des limites et contraintes évoquées par les médecins et l'influence que celles-ci peuvent avoir sur leur pratique, il semble difficile a priori que l'autonomie des patients soit garantie. En effet, comment garantir, en tant que soignant, l'aptitude au choix autonome du patient dans un contexte ou un dispositif (les DA) se trouve l'objet d'un dilemme personnel pour le médecin (légitimité, faisabilité, contraintes).

\section{CONCLUSION}

Ce type de recherche permet d'éclairer les enjeux psycho-sociaux présents dans la relation de soin à partir d'une méthodologie centrée sur l'expression des sentiments et représentations associés au dispositif des DA. Quelle que soit la pertinence supposée de ce dispositif, il est amené à se confronter à des limites d'ordre psychosocial qui représentent en partie des formes de « résistances » qui remettent en question sa " légitimité » ou " utilité ». Le développement des DA devra prendre en compte les enjeux que les différents acteurs (patients, soignants) assignent à ce dispositif et les croyances et représentations qu'ils développent à son égard.

\section{REFERENCES BIBLIOGRAPHIQUES}

1. Loi $n^{\circ} 2005-370$ du 22 avril 2005 relative aux droits des malades et à la fin de vie. Journal Officiel de la République Française du 23 avril 2005.

2. Décret $n^{\circ} 2016-1067$ du 3 août 2016 relatif aux directives anticipées prévues par la loi $n^{\circ} 2016-87$ du 2 février 2016 créant de nouveaux droits en faveur des malades et des personnes en fin de vie. Journal Officiel de la République Française du 5 août 2016

3. Loi $n^{\circ} 2016-87$ du 2 février 2016 créant de nouveaux droits en faveur des malades et des personnes en fin de vie. Journal Officiel de la République Française du 3 février 2016.

4. Sicard D. Penser solidairement la fin de vie. Commission de réflexion sur la fin de vie en France. Paris : La 
Documentation Française, 2012.

5. Guyon G, Garbacz L, Baumann A, Bohl, E, Maheut-Bosser A, Coudane H, Kanny G, Gillois P, Claudot, F. Personne de confiance et directives anticipées : défaut d'information et de mise en œuvre. Rev Med Interne $2014 ; 35: 643-648$

6. Pennec S, Monnier A, Pontone S, Aubry R. Les décisions médicales en fin de vie en France. Population \& Sociétés $2012 ; 494: 1-4$.

7. Cannone P, Baltayan C, Estienne L, Dany L, Tomasini P. Directives anticipées en France : représentations sociales et liberté d'utilisation. Psycho-Oncologie $2016 ; 10: 30-36$.

8. Cugliari AM, Miller T, Sobal, J. Factors promoting completion of advance directives in the hospital. Arch Int Med $1995 ; 15: 1893-1898$.

9. Eiser AR, Weiss MD. The underachieving advance directive: recommendations for increasing advance directive completion. Am J Bioeth $2001 ; 4$ : 10-17.

10. Halpern NA, Pastores SM, Chou JF, Chawla S, Thaler HT. Advance directives in an oncologic intensive care unit: a contemporary analysis of their frequency, type, and impact. J Palliat Med $2011 ; 14: 483-489$.

11. Hanson LC, Rodgman E. The use of living wills at the end of life. A national study. Arch Int Med $1996 ; 156$ : 1018-1022.

12. Patel RV, Sinuff T, Cook DJ. Influencing advance directive completion rates in non-terminally ill patients: a systematic review. J Critic Care $2004 ; 19: 1-9$.

13. Ditto PH, Hawkins NA. Advance directives and cancer decision making near the end of life. Health Psychol $2005 ; 24: 63-70$.

14. Blondeau D, Valois P, Keyserlingk EW, Hébert M, Lavoie M. Comparison of patients' and health care professionals' attitudes towards advance directives. J Med Ethics $1998 ; 24: 328-335$.

15. Hughes DL, Singer PA. Family physician's attitudes toward advance directives. CMAJ $1992 ; 146: 1937$ 1944.

16. Bond CJ, Lowton K. Geriatricians' view on advance decisions and their use in clinical care in England: qualitative study. Age Ageing $2011 ; 40: 450-456$.

17. Horn R. “I don't need my patients' opinion to withdraw treatment”: patient preferences at the end-of-life and physician attitudes towards advance directives in England and France. Med Health Care Philos $2014 ; 17$ : 425-435.

18. Dany L. Analyse qualitative du contenu des représentations sociales. In : Lo Monaco G, Delouvée S, Rateau P, eds. Les représentations sociales. Bruxelles : de Boeck, 2016 : 85-102.

19. Flick U. Thematic coding and content analysis. In : U. Flick, ed. An introduction to qualitative research. London: Sage, $2014: 420-438$

20. Mucchielli R. L'analyse de contenu des documents et des communications. Paris : ESF éditeurs, 1991. 
21. Ledoux M, Rhondali W, Monnin L, Thollet C, Gabon P, Filbet M. Directives anticipées: représentations d'infirmiers et de médecins en 2012. Bulletin du Cancer $2013 ; 100: 941-945$.

22. Dany L, Dudoit E, Salas S, Duffaud F. Les représentations des soins palliatifs. In : Saint-Jean M, Bastiani F, eds. L'épreuve de la fin de vie (pp. 143-164). Paris : Seli Arslan, 2014 : 143-164.

23. Morin M. Parcours de santé. Paris : Armand Colin, 2004.

24. Dow LA, Matsuyama RK, Ramakrishnan V, Kuhn L, Lamont EB, Lyckholm L, Smith TJ. Paradoxes in advance care planning: the complex relationship of oncology patients, their physicians, and advance medical directives. Journal of Clinical Oncology $2009 ; 28: 299-304$.

25. Periyakoil VS, Neri E, Fong A, Kraemer H. Do unto others: doctors' personal end-of-life resuscitation preferences and their attitudes toward advance directives. PloS one $2014 ; 9$ : e98246.

26. Carvais R, Sasportes M. La greffe humaine. Paris : PUF, 2000.

27. Vinant P, Rousseau I, Huillard O, Goldwasser F, Guillard MY, Colombet I. Respect des volontés en fin de vie : étude de faisabilité d'une information sur la personne de confiance et les directives anticipées. Bulletin du Cancer $2015 ; 102: 234-244$.

28. Wright AA, Zhang B, Ray A, Mack JW, Trice E, Balboni T, Mitchell SL, Jackson VA, Block SD, Maciejewski PK, Prigerson, HG. Associations between end-of-life discussions, patient mental health, medical care near death, and caregiver bereavement adjustment. Jama 2008 ; $300: 1665-1673$.

29. Beauchamp TL, Childress JF. Les principes de l'éthique biomédicale. Paris : Les Belles Lettres, 2008. 